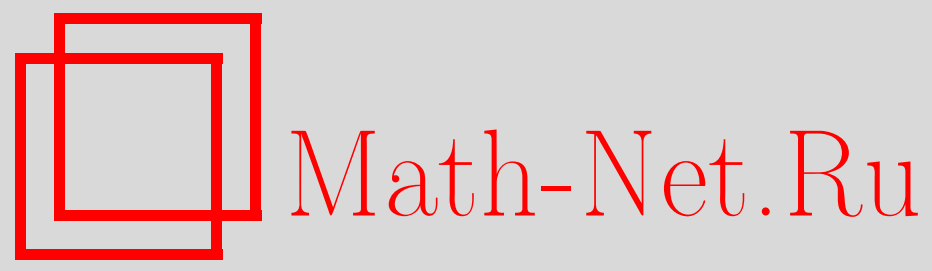

П. А. Пантелеев, Об отличимости состояний автоматов, Дискрет. матем., 2003, том 15, выпуск 3, 76-90

DOI: https://doi.org/10.4213/dm207

Использование Общероссийского математического портала Math-Net.Ru подразумевает, что вы прочитали и согласны с пользовательским соглашением http://www.mathnet.ru/rus/agreement

Параметры загрузки:

IP : 54.197 .130 .99

26 апреля 2023 г., 04:39:18 


\title{
Об отличимости состояний автоматов
}

\author{
(c) 2003 г. П. А. Пантелеев
}

\begin{abstract}
В статье исследуются различные варианты понятия отличимости, возникаюшие в результате расслоения классического понятия отличимости. Рассматривается отличимость в смысле заданной метрики на множестве выходных символов, $k$-отличимость и $\infty$-отличимость. Для каждого вида получено точное значение соответствующей функшии Шеннона. Найдено предельное значение параметра $k$, при котором из $k$-отличимости следует $\infty$-отличимость.

Работа выполнена при подлержке Российского фонда фундаментальных исследований, проект 02-01-00162.
\end{abstract}

\section{1. Введение}

Известная работа Э. Мура [1], приведшая к созданию теории экспериментов с автоматами, опиралась на явление отличимости состояний автомата, состоящее в том, что отличимые состояния по-разному реагируют на одно и то же входное слово. Это понятие допускает расслоение, которое здесь и исследуется.

В качестве первого варианта расслоения исследуются автоматы с заданной метрикой на множестве выходных символов. Отличимость здесь понимается как появление выходных символов, находящихся на расстоянии, большем, чем заданное. Получены точное значение функции Шеннона минимальной длины входного отличающего слова для автомата с $n$ состояниями и для состояний двух автоматов с $n$ и $m$ состояниями.

Второй подход основан на понятии $k$-отличимости, когда два состояния считаются $k$-отличимыми входным словом, если соответствующие выходные слова отличаются в $k$ позициях. Получено точное значение функции Шеннона минимальной длины $k$-отличающего слова для автомата с $n$ состояниями.

Естественным развитием понятия $k$-отличимости является понятие $\infty$-отличимости. Два состояния $\infty$-отличимы, если существует входное сверхслово такое, что соответствующие выходные сверхслова отличаются в бесконечном числе позиций. Получено точное значение функции Шеннона предельного значения параметра $k$, при котором из $k$-отличимости состояний автомата с $n$ состояниями следует их $\infty$-отличимость.

Задание метрики на множестве выходных символов эквивалентно заданию на нем произвольного рефлексивного и симметричного бинарного отношения $R$. В этом смысле можно говорить об $R$-отличимости состояний для произвольного отношения $R$. Третий вид отличимости основан на отношении $R$, для которого $x R y$ равносильно $x \neq y$. Здесь явно указан вид соответствующей функщии Шеннона. 


\section{2. Определения и результаты}

Под абстрактным конечным автоматом (в дальнейшем просто автоматом) будем понимать объект

$$
\mathfrak{A}=(A, Q, B, \varphi, \psi),
$$

где $A, Q, B$ - конечные непустые множества, называемые, соответственно, входным алфавитом, алфавитом состояний и выходным алфавитом, а $\varphi: Q \times A \rightarrow Q$ и $\psi: Q \times A \rightarrow B$ - функции переходов и выходов. Множество всех слов в алфавите $A$ обозначим $A^{*}$. Пусть $|\alpha|$ означает длину слова $\alpha \in A^{*}$. Если $\alpha=a(1) a(2) \ldots a(l)$, то обозначим символом $\left.\alpha\right|_{m}$ слово $a(1) a(2) \ldots a(m)$, где $1 \leqslant m \leqslant l$. Если $m=0$ и $\Lambda-$ пустое слово, то $\left.\alpha\right|_{m}=\Lambda$. Пусть $a \in A$, тогда полагаем, что $a^{n}$ обозначает слово $a \ldots a$ длины $n$. Аналогично, если $\alpha \in A^{*}$, то $[\alpha]^{n}$ означает слово $\alpha \ldots \alpha$ длины $n|\alpha|$. В обоих случаях считаем $a^{0}=[\alpha]^{0}=\Lambda$. Распространим функции $\varphi$ и $\psi$ на множество $Q \times A^{*}$, полагая

$$
\begin{array}{ll}
\varphi(q, \Lambda)=q, \quad \varphi(q, \alpha a)=\varphi(\varphi(q, \alpha), a), \\
\psi(q, \Lambda)=\Lambda, \quad \psi(q, \alpha a)=\psi(\varphi(q, \alpha), a), \quad q \in Q, \quad a \in A, \quad \alpha \in A^{*} .
\end{array}
$$

Пусть

$$
\bar{\psi}\left(q, \alpha^{l}\right)=\psi\left(q,\left.\alpha^{l}\right|_{1}\right) \psi\left(q,\left.\alpha^{l}\right|_{2}\right) \ldots \psi\left(q,\left.\alpha^{l}\right|_{l}\right) .
$$

Распространим функщию переходов $\varphi$ на множество $2^{Q} \times A^{*}$, где $2^{Q}=\left\{Q^{\prime} \mid Q^{\prime} \subseteq Q\right\}$, полагая

$$
\varphi\left(Q^{\prime}, \alpha\right)=\left\{\varphi(q, \alpha) \mid q \in Q^{\prime}\right\}
$$

где $\alpha \in A^{*}$ и $Q^{\prime} \subseteq Q$.

Обозначим через $K(A, B)$ класс всех автоматов с заданными входным $A$ и выходным $B$ алфавитами, а через $K_{n}(A, B)$ класс тех из них, которые имеют не более, чем $n$ состояний. Пусть $\mathfrak{A}_{1}, \mathfrak{A}_{2} \in K(A, B)$ и $q_{1}, q_{2}$ - их состояния. Назовем состояния $q_{1}$ и $q_{2}$ отличимыми, если существует входное слово $\alpha \in A^{*}$ такое, что $\psi_{1}\left(q_{1}, \alpha\right) \neq \psi_{2}\left(q_{2}, \alpha\right)$.

Зададим на множестве выходных символов $B$ метрику $\rho$. Возьмем произвольное число $\varepsilon>0$. Определим на множестве $B$ отношение $R$, следующим образом:

$$
x R y \Longleftrightarrow \rho(x, y) \leqslant \varepsilon .
$$

Про это отношение будем говорить, что оно определено метрикой $\rho$ и числом $\varepsilon$. Очевидно, что $R$ - рефлексивное и симметричное отношение. В то же время ясно, что задание такого отношения эквивалентно заданию произвольного рефлексивного и симметричного бинарного отношения на $B$. Поэтому, для упрощения дальнейшего изложения, везде далее на $B$ будем задавать не пару $\langle\rho, \varepsilon\rangle$, а указанное отношение $R$.

Обозначим через $\mathfrak{R}_{r}(B), \mathfrak{R}_{s}(B), \mathfrak{R}_{t}(B)$, соответственно, множества всех бинарных рефлексивных, симметричных и транзитивных отношений на $B$ и полагаем

$$
\mathfrak{R}_{r s}(B)=\mathfrak{R}_{r}(B) \cap \mathfrak{R}_{s}(B) .
$$

Пусть $R \in \mathfrak{R}_{r s}(B)$ и $\mathfrak{A}_{1}, \mathfrak{A}_{2} \in K(A, B)$. Назовем состояния $q_{1}$ и $q_{2}$ этих автоматов $R$-отличимыми словом $\alpha \in A^{*}$, если неверно, что $\psi_{1}\left(q_{1}, \alpha\right) R \psi_{2}\left(q_{2}, \alpha\right)$. Состояния $q_{1}$ и $q_{2}$ называются $R$-отличимыми, если существует входное слово, которое их $R$-отличает. В противном случае будем говорить, что они $R$-неотличимы. Пусть $L_{\mathfrak{Q}_{1}, \mathfrak{R}_{2}, R}\left(q_{1}, q_{2}\right)-$ минимальная длина входного слова, $R$-отличающего состояния $q_{1}$ и $q_{2}$ автоматов $\mathfrak{U}_{1}$ и $\mathfrak{A}_{2}$, и

$$
L_{R}\left(\mathfrak{U}_{1}, \mathfrak{A}_{2}\right)=\max _{q_{1}, q_{2}} L_{\mathfrak{A}_{1}, \mathfrak{I}_{2}, R}\left(q_{1}, q_{2}\right),
$$


где максимум берется по всем таким парам состояний. Если $\mathfrak{U}=\mathfrak{U}_{1}=\mathfrak{A}_{2}$, то вместо $L_{R}(\mathfrak{A}, \mathfrak{A})$ будем писать $L_{R}(\mathfrak{U})$. Определим функшии Шеннона $L_{R}(n)$ и $L_{R}(n, m)$, полагая

$$
\begin{aligned}
L_{R}(n) & =\max _{\mathfrak{A} \in K_{n}(A, B)} L_{R}(\mathfrak{U}), \\
L_{R}(n, m) & =\max _{\mathfrak{H}_{1} \in K_{n}(A, B), \mathfrak{A}_{2} \in K_{m}(A, B)} L_{R}\left(\mathfrak{U}_{1}, \mathfrak{U}_{2}\right),
\end{aligned}
$$

то есть $L_{R}(n)$ - минимальная длина $R$-отличаюшего слова в худшем случае на всех парах $R$-отличимых состояний $q_{1}$ и $q_{2}$ у всех автоматов $\mathfrak{A}$ с $n$ состояниями, а $L_{R}(n, m)-$ аналогичная функшия для двух автоматов с $n$ и $m$ состояниями. Заметим, что эти величины определены корректно, если $R \neq B \times B$, иначе у любого автомата не будет $R$-отличимых состояний. Поэтому в дальнейшем полагаем, что $R \neq B \times B$.

Лемма 1. Если $\mathfrak{A} \in K_{n}(A, B), R \in \Re_{r s}(B), q_{0}$ и $q_{0}^{\prime}-$ два произвольных $R$-отличимых состояния автомата $\mathfrak{A}$, то существует $\alpha \in A^{*}, R$-отличающее их и такое, что $|\alpha| \leqslant n(n-1) / 2$.

Доказательство. Пусть $\alpha=a(1) a(2) \ldots a(l)$ - кратчайшее слово, $R$-отличающее состояния $q_{0}$ и $q_{0}^{\prime}$. Определим две последовательности $q_{0}, q_{1}, \ldots, q_{l-1}$ и $q_{0}^{\prime}, q_{1}^{\prime}, \ldots, q_{l-1}^{\prime}$ следующим образом:

$$
q_{i}=\varphi\left(q_{0}, a(1) a(2) \ldots a(i)\right), \quad q_{i}^{\prime}=\varphi\left(q_{0}^{\prime}, a(1) a(2) \ldots a(i)\right), \quad i=1, \ldots, l-1 .
$$

В силу симметричности отношения $R$ можно говорить об отличимости неупорядоченной пары состояний $\left\{q, q^{\prime}\right\}$ словом $\alpha$, понимая под этим отличимость состояний $q$ и $q^{\prime}$. Легко видеть, что минимальная длина входного слова, $R$-отличающего неупорядоченную пару $\left\{q_{i}, q_{i}{ }^{\prime}\right\}$, есть $l-i$. Действительно, слово $a(i+1) a(i+2) \ldots a(l) R$-отличает состояния $q_{i}, q_{i}{ }^{\prime}$ и, если бы сушествовало слово $\beta,|\beta|<l-i$, также $R$-отличающее эти состояния, то слово $a(1) a(2) \ldots a(i) \beta R$-отличало бы состояния $q_{0}, q_{0}^{\prime}$ и имело бы длину, меньшую $l$. Это противоречит выбору слова $\alpha$. Очевидно, что все пары в последовательности $\left\{q_{0}, q_{0}^{\prime}\right\},\left\{q_{1}, q_{1}^{\prime}\right\}, \ldots,\left\{q_{l-1}, q_{l-1}^{\prime}\right\}$ различны, поскольку они имеют различные минимальные длины $R$-отличающих слов. Следовательно, $l$ не превосходит числа неупорядоченных пар элементов из множества $Q$, то есть $l \leqslant n(n-1) / 2$. Лемма доказана.

Теорема 1. Если $R \in \mathfrak{R}_{r s}(B)$, то

$$
L_{R}(n)= \begin{cases}n-1, & \text { если } R \in \mathfrak{R}_{t}(B), \\ n(n-1) / 2, & \text { если } R \notin \mathfrak{R}_{t}(B) .\end{cases}
$$

Доказательство. Пусть $R \in \mathfrak{R}_{r s}(B)$ и $\mathfrak{A} \in K_{n}(A, B)$. Если $R \in \mathfrak{R}_{t}(B)$, то оно является отношением эквивалентности и разбивает множество $B$ на классы эквивалентности $B_{1}, \ldots, B_{k}, k \geqslant 2$.

Рассмотрим автомат

$$
\widetilde{\mathfrak{A}}=(A, Q, \widetilde{B}, \varphi, \widetilde{\psi}),
$$

где $Q-$ множество состояний автомата $\mathfrak{A}, \widetilde{B}=\left\{B_{1}, \ldots, B_{k}\right\}$ и $\widetilde{\psi}(q, a)=B_{i}$, когда $\psi(q, a) \in B_{i}$. Очевидно, что состояния $q, q^{\prime}$ автомата $\mathfrak{A} R$-отличимы словом $\alpha$ точно тогда, когда они отличимы этим словом в автомате $\widetilde{\mathfrak{A}}$. Тогда согласно теореме Мура [1] минимальная длина отличающего слова в автомате $\widetilde{\mathfrak{U}}$ не больше $n-1$, и получаем неравенство $L_{R}(n) \leqslant n-1$. 


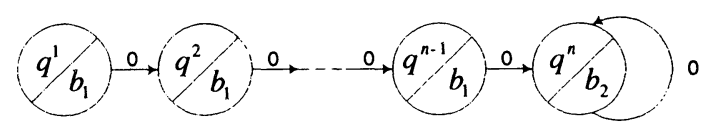

Рис. 1.

Рассмотрим автомат

$$
\mathfrak{U}=\left(\{0\},\left\{q^{1}, q^{2}, \ldots, q^{n}\right\}, B, \varphi, \psi\right)
$$

с заданным отношением эквивалентности $R$ на множестве его выходных символов, и пусть $b_{1} \neg R b_{2}$, где $b_{1}, b_{2} \in B$. Диаграмма Мура этого автомата изображена на рис. 1 . Из нее видно, что для состояний $q^{1}$ и $q^{2}$ минимальная длина отличающего слова равна $n-1$. Таким образом, мы доказали, что если $R \in \mathfrak{R}_{t}(B)$, то $L_{R}(n)=n-1$.

Пусть теперь $R \notin \mathfrak{R}_{t}(B)$. Установим, что $L_{R}(n)=n(n-1) / 2$. В силу леммы 1

$$
L_{R}(n) \leqslant n(n-1) / 2 \text {. }
$$

Докажем, что для $R \notin \Re_{t}(B)$ можно построить автомат

$$
\mathfrak{U} \in K_{n}(A, B),
$$

у которого существуют два $R$-отличимых состояния $q_{1}, q_{2}$ такие, что минимальная длина $R$-отличающего их слова равна $n(n-1) / 2$. Тем самым покажем, что $L_{R}(n)=n(n-1) / 2$. Для этого рассмотрим автомат

$$
\mathfrak{U}=(\{0,1\}, Q, B, \varphi, \psi)
$$

где $Q=\left\{q^{0}, q^{1}, \ldots, q^{n-1}\right\}$, функция выходов задана произвольно, а функция переходов определяется следующим образом:

$$
\varphi\left(q^{i}, 1\right)=q^{i+1} \quad(\bmod n), \quad \varphi\left(q^{n-1}, 0\right)=q^{0}, \quad \varphi\left(q^{i}, 0\right)=q^{i}, \quad i \neq n-1 .
$$

Этот автомат был независимо предложен А. Е. Кирнасовым для получения нижней оценки функции Шеннона длины склеивающего слова. Диаграмма Мура для этого автомата приведена на рис. 2.

Упорядочим всевозможные пары состояний автомата $\mathfrak{A}$. Пусть

$$
Q_{s}^{r}=\left\{q^{s}, q^{s+k-r} \quad(\bmod n)\right\},
$$

где $n=2 k$ в случае четного $n$, и $n=2 k+1$ в случае нечетного, $k=[n / 2]$.

Если $n=2 k$, то порядок следующий:

$$
\begin{aligned}
Q_{0}^{0} & =\left\{q^{0}, q^{k}\right\}, \dot{Q}_{1}^{0}=\left\{q^{1}, q^{k+1}\right\}, \ldots, Q_{k-1}^{0}=\left\{q^{k-1}, q^{n-1}\right\}, \\
Q_{0}^{1} & =\left\{q^{0}, q^{k-1}\right\}, Q_{1}^{1}=\left\{q^{1}, q^{k}\right\}, \ldots, Q_{n-1}^{1}=\left\{q^{n-1}, q^{k-2}\right\}, \\
Q_{0}^{2} & =\left\{q^{0}, q^{k-2}\right\}, Q_{1}^{2}=\left\{q^{1}, q^{k-1}\right\}, \ldots, Q_{n-1}^{2}=\left\{q^{n-1}, q^{k-3}\right\}, \\
& \ldots \\
Q_{0}^{k-1} & =\left\{q^{0}, q^{1}\right\}, Q_{1}^{k-1}=\left\{q^{1}, q^{2}\right\}, \ldots, Q_{n-1}^{k-1}=\left\{q^{n-1}, q^{0}\right\} .
\end{aligned}
$$




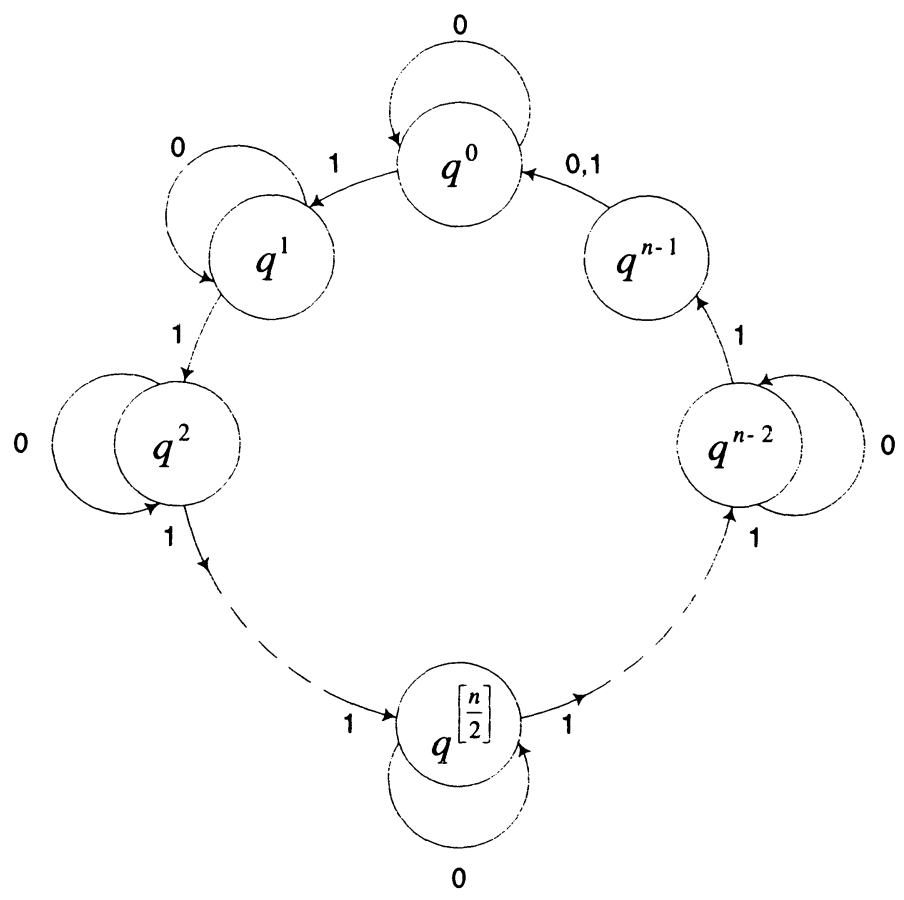

Рис. 2.

Если $n=2 k+1$, то порядок следующий:

$$
\begin{aligned}
Q_{0}^{0} & =\left\{q^{0}, q^{k}\right\}, Q_{1}^{0}=\left\{q^{1}, q^{k+1}\right\}, \ldots, Q_{n-1}^{0}=\left\{q^{n-1}, q^{k-1}\right\}, \\
Q_{0}^{1} & =\left\{q^{0}, q^{k-1}\right\}, Q_{1}^{1}=\left\{q^{1}, q^{k}\right\}, \ldots, Q_{n-1}^{1}=\left\{q^{n-1}, q^{k-2}\right\}, \\
& \ldots \\
Q_{0}^{k-1} & =\left\{q^{0}, q^{1}\right\}, Q_{1}^{k-1}=\left\{q^{1}, q^{2}\right\}, \ldots, Q_{n-1}^{k-1}=\left\{q^{n-1}, q^{0}\right\} .
\end{aligned}
$$

Рассмотрим таблицу 1.

Пусть $v(\widetilde{Q})$ означает номер пары состояний $\widetilde{Q}$ в введенном упорядочении. Тогда из таблицы 1 видно, что входным символом склеивается только пара состояний, стоящая последней в введенном порядке: $\varphi\left(\left\{q^{0}, q^{n-1}\right\}, 0\right)=\left\{q^{0}\right\}$. Далее, если $\widetilde{Q}^{\prime}=\varphi(\widetilde{Q}, a)$, то из таблицы 1 легко видеть, что $v\left(\widetilde{Q}^{\prime}\right) \leqslant v(\widetilde{Q})+1$, то есть под действием любого входного символа $a \in A$ пара состояний $\widetilde{Q}$ переходит в пару состояний $\widetilde{Q}^{\prime}$, стоящую в введенном порядке не более чем на один элемент впереди. Отсюда заключаем, что если $\varphi\left(\left\{q^{0}, q^{[n / 2]}\right\}, \alpha\right)=\left\{q^{0}, q^{n-1}\right\}$ для некоторого $\alpha \in A^{*}$, то, поскольку $v\left(\left\{q^{0}, q^{[n / 2]}\right\}\right)=1$ и $v\left(\left\{q^{0}, q^{n-1}\right\}\right)=n(n-1 / 2$, справедливо неравенство $|\alpha| \leqslant n(n-1) / 2-1$. Следовательно, если $\beta$ из $A^{*}$ склеивает пару состояний $\left\{q^{0}, q^{[n / 2]}\right\}$, то у него существует начало $\beta^{\prime}$ такое, что

$$
\varphi\left(\left\{q^{0}, q^{[n / 2]}\right\}, \beta^{\prime}\right)=\left\{q^{0}, q^{n-1}\right\},
$$

где $\left|\beta^{\prime}\right| \leqslant n(n-1) / 2-1$ и поэтому $|\beta| \leqslant n(n-1) / 2$. Входное слово $\gamma=1^{k-1} 0\left[1^{n-1} 0\right]^{k-1}$, если $n=2 k$ или $\gamma=\left[1^{n-1} 0\right]^{k}$, если $n=2 k+1$, имеет длину $n(n-1) / 2$ и склеивает 


\section{Таблица 1.}

\begin{tabular}{|c|c|c||c|c|c|}
\hline \multicolumn{2}{|c|}{$n=2 k$} & \multicolumn{3}{c|}{$n=2 k+1$} \\
\hline$Q$ & $\varphi(Q, 0)$ & $\varphi(Q, 1)$ & \multicolumn{1}{c|}{$Q$} & $\varphi(Q, 0)$ & $\varphi(Q, 1)$ \\
\hline$Q_{k-1}^{0}=\left\{q^{k-1}, q^{n-1}\right\}$ & $Q_{0}^{1}$ & $Q_{0}^{0}$ & $Q_{k}^{0}=\left\{q^{k}, q^{n-1}\right\}$ & $Q_{0}^{0}$ & $Q_{k+1}^{0}$ \\
\hline$Q_{n-1}^{k-1}=\left\{q^{n-1}, q^{0}\right\}$ & $\left\{q^{0}\right\}$ & $Q_{0}^{k-1}$ & $Q_{n-1}^{k-1}=\left\{q^{n-1}, q^{0}\right\}$ & $\left\{q^{0}\right\}$ & $Q_{0}^{k-1}$ \\
\hline $\begin{array}{l}Q_{n-1}^{r}=\left\{q^{n-1}, q^{k-r-1}\right\} ; \\
0<r<k-1\end{array}$ & $Q_{0}^{r+1}$ & $Q_{0}^{r}$ & $\begin{array}{l}Q_{n-1}^{r}=\left\{q^{n-1}, q^{k-r-1}\right\} ; \\
0<r<k-1\end{array}$ & $Q_{0}^{r+1}$ & $Q_{0}^{r}$ \\
\hline $\begin{array}{l}Q_{k+r-1}^{r}=\left\{q^{k+r-1}, q^{n-1}\right\} ; \\
0<r<n-k\end{array}$ & $Q_{k+r-1}^{r-1}$ & $Q_{k+r}^{r}$ & $\begin{array}{l}Q_{k+r}^{r}=\left\{q^{k+r}, q^{n-1}\right\} ; \\
0<r<n-k-1\end{array}$ & $Q_{k+r}^{r-1}$ & $Q_{k+r+1}^{r}$ \\
\hline $\begin{array}{l}Q_{s}^{r}, s \neq n-1, k+r-1 ; \\
n-1 \notin Q_{s}^{r}\end{array}$ & $Q_{s}^{r}$ & $Q_{s+1}^{r}$ & $\begin{array}{l}Q_{s}^{r}, s \neq n-1, k+r ; \\
n-1 \notin Q_{s}^{r}\end{array}$ & $Q_{s}^{r}$ & $Q_{s+1}^{r}$ \\
\hline
\end{tabular}

состояния $q^{0}$ и $q^{[n / 2]}$, а слово $\gamma^{\prime}=\left.\gamma\right|_{|\gamma|-1}$ длины $n(n-1) / 2-1$ переводит эти состояния в состояния $q^{0}$ и $q^{n-1}$, соответственно. Тем самым, учитывая полученные ранее неравенства, мы доказали, что минимальная длина слова, склеивающего состояния $q^{0}$ и $q^{[n / 2]}$, равна $n(n-1) / 2$, а минимальная длина слова, переводящего эти состояния в состояния $q^{0}$ и $q^{n-1}$, равна $n(n-1) / 2-1$.

Пусть $R \in \Re_{r s}(B)$, но $R \notin \Re_{t}(B)$. Тогда должны существовать выходные символы $b_{1}, b_{2}, b_{3} \in B$ такие, что $b_{1} R b_{2}$ и $b_{2} R b_{3}$, но $b_{1} \neg R b_{3}$. Определим функцию выходов автомата $\mathfrak{A}$ следуюшим образом: $\psi\left(q^{0}, a\right)=b^{1}, \psi\left(q^{n-1}, a\right)=b^{3}$ и $\psi\left(q^{i}, a\right)=b^{2}$ при $i \neq 0, n-1$, $a \in A$. Легко видеть, что слово $\alpha^{l} \in A^{*} R$-отличает состояния $q_{1}$ и $q_{2}$ точно тогда, когда $\varphi\left(\left\{q_{1}, q_{2}\right\},\left.\alpha^{l}\right|_{l-1}\right)=\left\{q^{0}, q^{n-1}\right\}$. Тогда из доказанного выше следует, что минимальная длина $R$-отличающего состояния $q^{0}$ и $q^{[n / 2]}$ слова равна $n(n-1) / 2-1+1=n(n-1) / 2$. Окончательно получаем для $\mathfrak{A}$ равенство

$$
L_{\mathfrak{Y}, R}\left(q^{0}, q^{[n / 2]}\right)=n(n-1) / 2,
$$

что и требовалось доказать.

Лемма 2. Если $\mathfrak{P}_{1} \in K_{n}(A, B), \mathfrak{U}_{2} \in K_{m}(A, B) u R \in \mathfrak{R}_{r s}(B)$, то длина кратчайшего входного слова $\alpha$, R-отличающего состояния $q_{0}$, $q_{0}^{\prime}$ автоматов $\mathfrak{A}_{1}, \mathfrak{U}_{2}$, не превосходит $n m$.

Доказательство. Пусть $\alpha=a(1) a(2) \ldots a(l)$ - такое слово. Определим две последовательности $q_{0}, q_{1}, \ldots, q_{l-1}$ и $q_{0}{ }^{\prime}, q^{\prime}{ }^{\prime}, \ldots, q_{l-1}{ }^{\prime}$ следующим образом:

$$
q_{i}=\varphi_{1}\left(q_{0}, a(1) a(2) \ldots a(i)\right), \quad q_{i}^{\prime}=\varphi_{2}\left(q_{0}^{\prime}, a(1) a(2) \ldots a(i)\right), \quad i=1, \ldots, l-1 .
$$

Заметим, что минимальная длина входного слова, $R$-отличающего состояния $q_{i} \in Q_{1}$ и $q_{i}{ }^{\prime} \in Q_{2}$ есть $l-i$. Действительно, слово $a(i+1) a(i+2) \ldots a(l) R$-отличает состояния $q_{i}, q_{i}{ }^{\prime}$ и, если бы существовало слово $\beta,|\beta|<l-i$, также $R$-отличающее эти состояния, то слово $a(1) a(2) \ldots a(i) \beta$ также $R$-отличало состояния $q_{0}, q_{0}^{\prime}$ и имело длину, меньшую $l$. Это противоречит выбору $\alpha$. Очевидно, что все пары в последовательности $\left(q_{0}, q_{0}^{\prime}\right),\left(q_{1}, q_{1}^{\prime}\right), \ldots,\left(q_{l-1}, q_{l-1}\right)$ различны, поскольку они имеют различные минимальные длины $R$-отличающих слов. Следовательно, $l$ не превосходит числа упорядоченных пар из множества $Q_{1} \times Q_{2}$, то есть $l \leqslant n m$. Лемма доказана. 


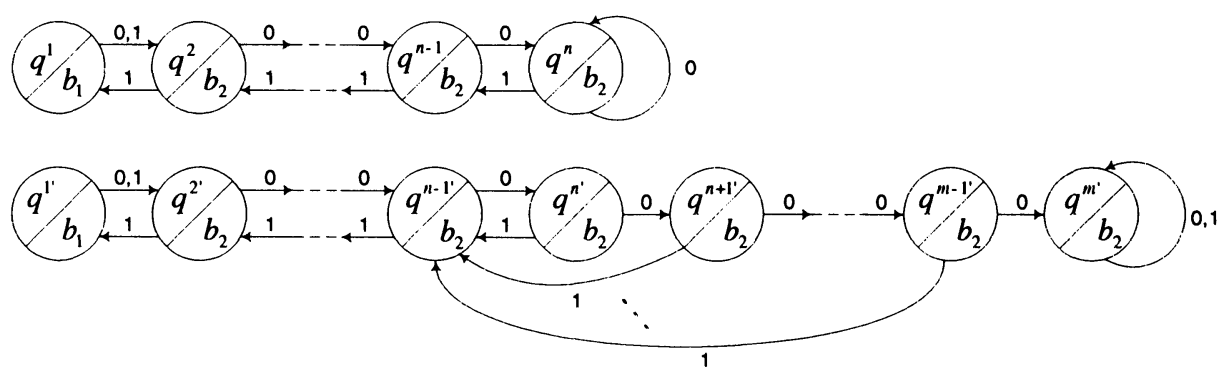

Рис. 3.

Теорема 2. Пусть $R \in \mathfrak{R}_{r s}(B)$, тогда

$L_{R}(n, m)=n+m-1$, если $R \in \Re_{t}(B) ;$

$\max _{1 \leqslant k \leqslant n, 1 \leqslant l \leqslant m .(k . l)=1} k l \leqslant L_{R}(n, m) \leqslant n m$, если $R \notin \Re_{t}(B) ;$

$L_{R}(n, m)=n m$, если $R \notin \Re_{t}(B)$ и $n$ делит $m$ или наоборот.

Доказательство. В случае, когда отношение $R$ транзитивно, оно является эквивалентностью и разбивает $B$ на классы эквивалентности $B_{1}, \ldots, B_{k}, k \geqslant 2$. Рассмотрим автоматы $\mathfrak{A}_{1} \in K_{n}(A, B)$ и $\mathfrak{U}_{2} \in K_{m}(A, B)$. Аналогично тому, как это делалось при доказательстве теоремы 1 , построим автоматы $\widetilde{\mathfrak{A}}_{1}$ и $\widetilde{\mathfrak{A}}_{2}$. Очевидно, что слово $\alpha R$-отличает состояния $q_{1}$ и $q_{2}$ автоматов $\mathfrak{A}_{1}$ и $\mathfrak{A}_{2}$ точно тогда, когда оно отличает эти состояния в автоматах $\mathfrak{A}_{1}$ и $\mathfrak{A}_{2}$. Согласно теореме Мура (см. [1]), минимальная длина слова, отличающего состояния $q_{1}$ и $q_{2}$, для двух автоматов с $n$ и $m$ состояниями не превосходит $n+m-1$, таким образом, мы доказали, что в случае транзитивности $R$ выполняется неравенство $L_{R}(n, m) \leqslant n+m-1$. С другой стороны, следуюший пример показывает, что эта оценка не может быть понижена. Действительно, рассмотрим автоматы

$$
\mathfrak{A}=\left(\{0,1\},\left\{q^{1}, q^{2}, \ldots, q^{n}\right\}, B, \varphi, \psi\right), \quad \mathfrak{B}=\left(\{0,1\},\left\{q^{1^{\prime}}, q^{2^{\prime}}, \ldots, q^{m^{\prime}}\right\}, B, \varphi^{\prime}, \psi^{\prime}\right),
$$

диаграммы Мура которых приведены на рис. 3. Возьмем $b_{1} \in B_{1}, b_{2} \in B_{2}$.

Пусть $R \notin \Re_{t}(B)$. Из леммы 2 вытекает, что $L_{R}(n, m) \leqslant n m$. Покажем, что

$$
\max _{1 \leqslant l \leqslant n, 1 \leqslant k \leqslant m,(l, k)=1} l k \leqslant L_{R}(n, m) .
$$

Действительно, пусть $l_{0}, k_{0}$ таковы, что $1 \leqslant l_{0} \leqslant n, 1 \leqslant k_{0} \leqslant m,\left(l_{0}, k_{0}\right)=1$ и

$$
l_{0} k_{0}=\max _{1 \leqslant l \leqslant n, 1 \leqslant k \leqslant m,(l, k)=1} l k .
$$

Тогда рассмотрим автоматы $\mathfrak{A}=(A, Q, B, \varphi, \psi)$ и $\mathfrak{B}=\left(A, Q^{\prime}, B, \varphi^{\prime}, \psi^{\prime}\right)$, где $A=\{0\}, Q=\left\{q^{0}, q^{1}, \ldots, q^{n-1}\right\}, Q^{\prime}=\left\{q^{0^{\prime}}, q^{1^{\prime}}, \ldots, q^{m-1^{\prime}}\right\}$. Поскольку $R$ не транзитивно, существуют выходные символы $b_{1}, b_{2}, b_{3}$ такие, что $b_{1} R b_{2}$ и $b_{2} R b_{3}$, но $b_{1} \neg R b_{3}$. 

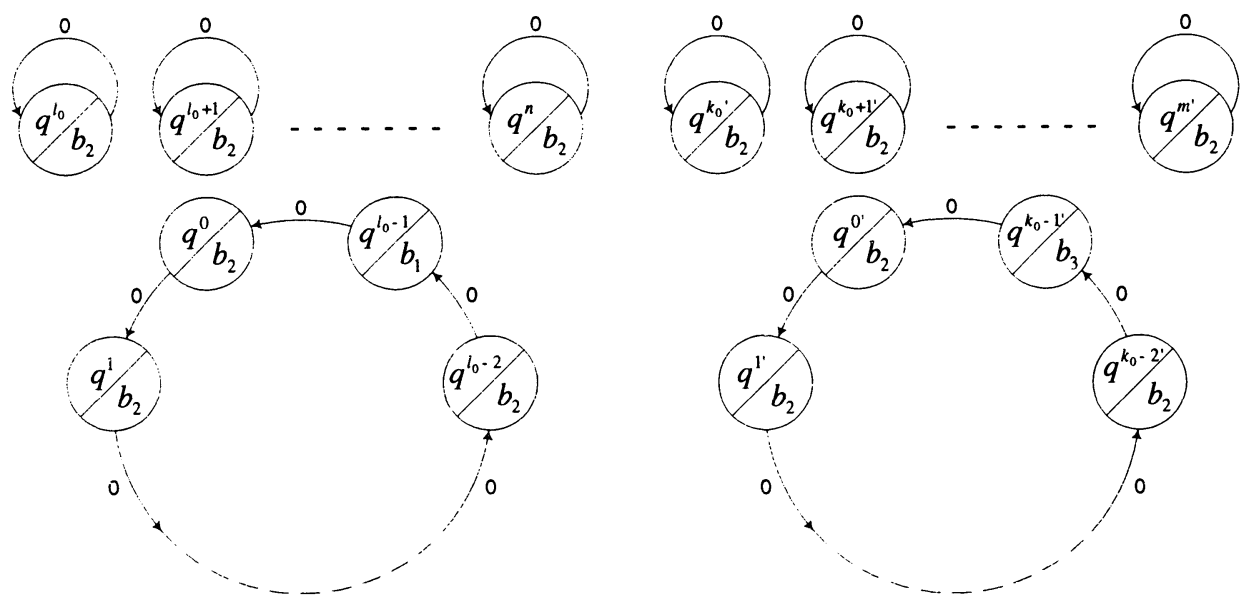

Рис. 4.

Функщии переходов и выходов зададим так:

$$
\begin{aligned}
& \varphi\left(q^{i}, 0\right)= \begin{cases}q^{i+1}\left(\bmod l_{0}\right) & \text { при } i=0,1, \ldots, l_{0}-1, \\
q^{i} & \text { при } i=l_{0}, l_{0}+1, \ldots, n-1,\end{cases} \\
& \varphi^{\prime}\left(q^{i^{\prime}}, 0\right)= \begin{cases}q^{i+1}\left(\bmod k_{0}\right)^{\prime} & \text { при } i=0,1, \ldots, k_{0}-1, \\
q^{i^{\prime}} & \text { при } i=k_{0}, k_{0}+1, \ldots, m-1,\end{cases} \\
& \psi\left(q^{i}, 0\right)= \begin{cases}b_{1} & \text { при } i=l_{0}-1, \\
b_{2} & \text { в остальных случаях }\end{cases} \\
& \psi^{\prime}\left(q^{i^{\prime}}, 0\right)= \begin{cases}b_{3} & \text { при } i=k_{0}-1 \\
b_{2} & \text { в остальных случаях. }\end{cases}
\end{aligned}
$$

Диаграммы состояний этих автоматов изображены на рис. 4. Положим $\alpha=0^{s}$. Пусть $x \mid y$ обозначает, что $x$ делит $y$. Ясно, что $\psi\left(q^{0}, \alpha\right)=b^{1}$ точно тогда, когда $l_{0} \mid s$. И аналогично

$$
\psi^{\prime}\left(q^{0^{\prime}}, \alpha\right)=b^{3} \Longleftrightarrow k_{0} \mid s .
$$

Очевидно, наименьшее такое $s$ равно наименьшему общему кратному $l_{0}$ и $k_{0}$. Но поскольку наибольший общий делитель $\left(l_{0}, k_{0}\right)=1$, то $s=l_{0} k_{0}$. Понятно, что слово $\alpha R$-отличает состояния $q^{0}$ и $q^{0^{\prime}}$ точно тогда, когда $\psi\left(q^{0}, \alpha\right)=b^{1}$ и $\psi^{\prime}\left(q^{0^{\prime}}, \alpha\right)=b^{3}$. Следовательно, минимальная длина $R$-отличающего эти состояния слова равна

$$
l_{0} k_{0}=\max _{1 \leqslant l \leqslant n, 1 \leqslant k \leqslant m,(l, k)=1} l k .
$$

Таким образом,

$$
L_{R}(n, m) \geqslant \max _{1 \leqslant l \leqslant n, 1 \leqslant k \leqslant m,(l, k)=1} l k .
$$

Теперь докажем, что если $n \mid m$ или $m \mid n$, то $L_{R}(n, m)=n m$. Без ограничения общности будем считать, что $m=n k$, где $k \in \mathbf{N}$. Рассмотрим два автомата

$$
\mathfrak{U}=(A, Q, B, \varphi, \psi), \quad \mathfrak{B}=\left(A, Q^{\prime}, B, \varphi^{\prime}, \psi^{\prime}\right),
$$



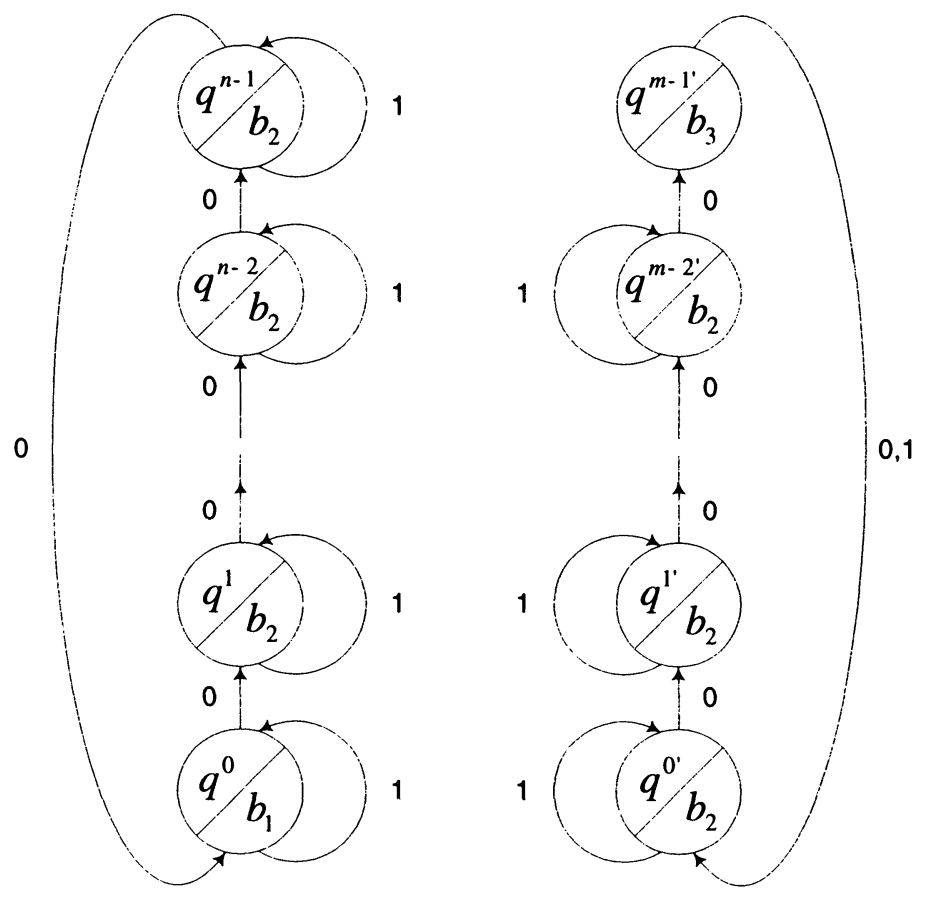

Рис. 5.

где $A=\{0,1\}, Q=\left\{q^{0}, q^{1}, \ldots, q^{n-1}\right\}, Q^{\prime}=\left\{q^{0^{\prime}}, q^{1^{\prime}}, \ldots, q^{m-1^{\prime}}\right\}$. Диаграммы состояний этих автоматов изображены на рис. 5 .

Пусть $d(a, b)=b-a(\bmod n)$. Определим линейный порядок на множестве $Q \times Q^{\prime}$, полагая

$\left(q^{i_{1}}, q^{j_{1}{ }^{\prime}}\right) \preccurlyeq\left(q^{i_{2}}, q^{j_{2}{ }^{\prime}}\right) \Longleftrightarrow d\left(i_{1}, j_{1}\right) \leqslant d\left(i_{2}, j_{2}\right)$ или $d\left(i_{1}, j_{1}\right)=d\left(i_{2}, j_{2}\right), \quad j_{1}<j_{2}$.

Занумеруем элементы множества $Q \times Q^{\prime}$ по возрастанию в смысле введенного порядка:

$$
\begin{aligned}
c_{0} & =\left(q^{0}, q^{0^{\prime}}\right), c_{1}=\left(q^{1}, q^{1^{\prime}}\right), \ldots, c_{i}=\left(q^{i}(\bmod n), q^{i^{\prime}}\right), \ldots, c_{m-1}=\left(q^{n-1}, q^{m-1^{\prime}}\right), \\
c_{m} & =\left(q^{n-1}, q^{0^{\prime}}\right), c_{m+1}=\left(q^{0}, q^{1^{\prime}}\right), \ldots, c_{m+j}=\left(q^{j-1}(\bmod n), q^{j^{\prime}}\right), \ldots, \\
c_{n m-2} & =\left(q^{n-1}, q^{m-2^{\prime}}\right), c_{n m-1}=\left(q^{0}, q^{m-1^{\prime}}\right)
\end{aligned}
$$

Пусть $v\left(c_{i}\right)=i$. Покажем, что для любых состояний $q^{i_{1}}$ и $q^{j_{1}^{\prime}}$ и входного символа $a$ справедливо неравенство

$$
v\left(\varphi\left(q^{i_{1}}, a\right), \varphi^{\prime}\left(q^{j_{1}^{\prime}}, a\right)\right)-v\left(q^{i_{1}}, q^{j_{1}{ }^{\prime}}\right) \leqslant 1 .
$$

Действительно, пусть $j_{1}<m-1$. Тогда $\varphi\left(q^{i_{1}}, 1\right)=q^{i_{1}}$ и $\varphi^{\prime}\left(q^{j_{1}}, 1\right)=q^{j_{1}{ }^{\prime}}$. Аналогично $\varphi\left(q^{i_{1}}, 0\right)=q^{i_{2}}$, где $i_{2}=i_{1}+1(\bmod n)$ и $\varphi^{\prime}\left(q^{j_{1}{ }^{\prime}}, 0\right)=q^{j_{2}{ }^{\prime}}$, где $j_{2}=j_{1}+1$. Поэтому в обоих случаях для любого $a \in\{0,1\}$

$$
v\left(\varphi\left(q^{i_{1}}, a\right), \varphi^{\prime}\left(q^{j_{1}^{\prime}}, a\right)\right)-v\left(q^{i_{1}}, q^{j_{1}{ }^{\prime}}\right) \leqslant 1 .
$$


Предположим теперь, что $j_{1}=m-1$, тогда $\varphi\left(q^{i_{1}}, 0\right)=q^{i_{2}}$, где $i_{2}=i_{1}+1(\bmod n)$ и $\varphi^{\prime}\left(q^{m-1^{\prime}}, 0\right)=q^{0^{\prime}}$. Очевидно, что $d\left(i_{1}, m-1\right)=d\left(i_{2}, 0\right)$ и $\left(q^{i_{1}}, q^{m-1^{\prime}}\right) \succcurlyeq\left(q^{i_{2}}, q^{0^{\prime}}\right)$. Далее, $\varphi\left(q^{i_{1}}, 1\right)=q^{i_{1}}, \varphi^{\prime}\left(q^{m-1^{\prime}}, 1\right)=q^{0^{\prime}}$. Ясно, что в обоих случаях выполняется неравенство $v\left(\varphi\left(q^{i}, a\right), \varphi^{\prime}\left(q^{j}, a\right)\right)-v\left(q^{i}, q^{j^{\prime}}\right) \leqslant 1$ для любого $a \in\{0,1\}$. Тогда получаем, что длина слова $\alpha$ такого, что $\varphi\left(q^{0}, \alpha\right)=q^{0}$ и $\varphi^{\prime}\left(q^{0^{\prime}}, \alpha\right)=q^{m-1^{\prime}}$ не менее, чем $v\left(q^{0}, q^{m-1^{\prime}}\right)-v\left(q^{0}, q^{0^{\prime}}\right)=n m-1$. Поэтому минимальная длина входного слова, $R$-отличающего состояния $q^{0}, q^{0^{\prime}}$, не менее, чем $n m$ и $L_{R}(n, m)=n m$. Таким образом, теорема 2 полностью доказана.

Пусть $\alpha=a(1) \ldots a(l), \beta=b(1) \ldots b(l)$. Обозначим через $\rho(\alpha, \beta)$ число позиций $i \in\{1,2, \ldots, l\}$, в которых $a(i) \neq b(i)$.

Назовем состояния $q_{1}, q_{2}$ автомата $\mathfrak{U} k$-отличимыми словом $\alpha$, если

$$
\rho\left(\bar{\psi}\left(q_{1}, \alpha\right), \bar{\psi}\left(q_{2}, \alpha\right)\right)=k
$$

то есть соответствуюшие выходные слова отличаются ровно в $k$ позициях. Будем говорить, что два состояния $k$-отличимы, если сушествует слово, которое их $k$-отличает.

Пусть $L_{\mathfrak{Q}}^{k}\left(q_{1}, q_{2}\right)$ - минимальная длина слова, $k$-отличающего состояния $q_{1}, q_{2}$ и $L^{k}(\mathfrak{U})$ - максимальное значение $L_{\mathfrak{l}}^{k}\left(q_{1}, q_{2}\right)$ на всех парах $k$-отличимых состояния автомата $\mathfrak{A}$. Рассмотрим функцию Шеннона

$$
L(n, k)=\max _{\mathfrak{U} \in K_{n}(A, B)} L^{k}(\mathfrak{U}) .
$$

Теорема 3. Справедливо равенство

$$
L(n, k)=n-1+(k-1) \frac{n(n-1)}{2} .
$$

Доказательство. Покажем индукцией по $k$, что для автомата $\mathfrak{A} \in K_{n}(A, B)$ и для любых его двух $k$-отличимых состояний $q_{1}, q_{2}$ существует слово $\alpha, k$-отличающее их, с длиной

$$
|\alpha| \leqslant n-1+(k-1) \frac{n(n-1)}{2} .
$$

При $k=1$ это следует из теоремы Мура. Допустим, что утверждение справедливо при произвольном $k$. Докажем его для $k+1$.

Пусть $q_{1}, q_{2}-(k+1)$-отличимые состояния автомата $\mathfrak{A}$ и $\alpha$-кратчайшее слово, $(k+1)$ отличающее эти состояния. У этого слова есть собственное начало $\beta^{l}=b(1) b(2) \ldots b(l)$ (то есть $\alpha=\beta^{l} \gamma$ ), которое отличает $q_{1}, q_{2}$ и переводит их в отличимые состояния $q_{1}{ }^{\prime}, q_{2}{ }^{\prime}$ $($ так как $k+1 \geqslant 2$ ). Рассмотрим последовательность пар состояний автомата $\mathfrak{U}$

$$
Q_{0}, Q_{1}, \ldots, Q_{l-1}
$$

где $Q_{0}=\left\{q_{1}, q_{2}\right\}, Q_{i}=\varphi\left(Q_{0}, \beta^{i}\right), \beta^{i}=b(1) b(2) \ldots b(i), i=1,2, \ldots, l-1$. Очевидно, что пара состояний $Q_{i}$ неотличима никаким словом $\delta$ таким, что

$$
\varphi\left(Q_{0}, \delta\right)=Q_{l-1}=\left\{q_{1}^{\prime}, q_{2}^{\prime}\right\}, \quad|\delta|<l-i,
$$

поскольку в противном случае слово $\beta^{i} \delta \gamma(k+1)$-отличает состояния $q_{1}$ и $q_{2}$, что противоречит выбору $\alpha$. Поэтому все элементы $Q_{i}$ различны, а это означает, что $l$ не превосходит числа всех возможных двухэлементных подмножеств $n$-элементного множества $Q$, то 
есть $\left|\beta^{l}\right|=l \leqslant n(n-1) / 2$. Поскольку $\alpha$ минимально по длине, $\gamma$ - тоже минимальное по длине слово, $k$-отличающее состояния $q_{1}{ }^{\prime}, q_{2}{ }^{\prime}$, и по предположению индукции $|\gamma| \leqslant n-1+(k-1) n(n-1) / 2$. Итак,

$$
|\alpha|=\left|\beta^{l}\right|+|\gamma| \leqslant n-1+k \frac{n(n-1)}{2},
$$

что и требовалось доказать.

Таким образом, мы доказали, что справедливо неравенство

$$
L(n, k) \leqslant n-1+(k-1) n(n-1) / 2 .
$$

Покажем, что эта оценка не понижаема. Рассмотрим автомат $\mathfrak{A}=(A, Q, B, \varphi, \psi)$, где $A=\{0,1, \ldots, n\}, Q=\left\{q^{1}, q^{2}, \ldots, q^{n}\right\}, B=\{0,1\}$, а функции переходов и выходов определены так:

$$
\begin{aligned}
\psi\left(q^{i}, a\right) & = \begin{cases}1, & \text { если } i=n, a=0, \\
0 & \text { в противном случае, }\end{cases} \\
\varphi\left(q^{i}, 0\right) & = \begin{cases}q^{2}, & \text { если } i=n-1, \\
q^{1}, & \text { если } i \neq n-1 .\end{cases}
\end{aligned}
$$

Если $a \neq 0$, то при $i<n$

$$
\varphi\left(q^{i}, a\right)= \begin{cases}q^{i+1}, & \text { если } a=i, \\ q^{a}, & \text { если } a<i, \\ q^{i}, & \text { если } a>i,\end{cases}
$$

и при $i=n$

$$
\varphi\left(q^{n}, a\right)= \begin{cases}q^{a+2}, & \text { если } a<n-1, \\ q^{n}, & \text { если } a \geqslant n-1 .\end{cases}
$$

Докажем индукцией по $k$, что если слово $\alpha k$-отличает состояния $q^{1}, q^{2}$, то

$$
|\alpha| \geqslant n-1+(k-1) \frac{n(n-1)}{2} .
$$

Действительно, при $k=1$ это следует из определения функций переходов и выходов автомата $\mathfrak{A}$. Допустим, что утверждение справедливо при произвольном $k$. Докажем его для $k+1$. Пусть $\alpha$ - минимальное по длине слово, $(k+1)$-отличающее $q^{1}, q^{2}$. Рассмотрим кратчайшее собственное начало $\beta^{l}$ слова $\alpha=\beta^{l} \gamma$, отличающее эти состояния и не склеивающее их. Оно существует, поскольку $k+1 \geqslant 2$ и состояния $q^{1}$ и $q^{2}$ хотя бы 2-олличимы. Поэтому, очевидно, одно из слов $\bar{\psi}\left(q^{l}, \beta^{l}\right)$ и $\bar{\psi}\left(q^{2}, \beta^{l}\right)$ оканчивается на 1. Отсюда и из определения функций выходов и переходов автомата $\mathfrak{U}$ получаем, что слово $\beta^{l}$ оканчивается на 0 и $q^{n} \in \varphi\left(\left\{q^{1}, q^{2}\right\},\left.\beta^{l}\right|_{l-1}\right)$ (так как только находясь в состоянии $q^{n}$ и. подавая на вход 0 , можно получить на выходе 1). Поскольку $\varphi\left(q^{n}, 0\right)=q^{1}$, второе состояние, входящее в $Q^{\prime}$, есть $q^{n-1}$ (так как $\varphi\left(q^{n-1}, 0\right)=q^{2} \neq q^{1}$, а для всех остальных состояний $\varphi\left(q^{i}, 0\right)=q^{1}, i \neq n-1$, то есть $\beta^{l}$ склеивает состояния $q^{1}, q^{2}$, что противоречит выбору $\left.\beta^{l}\right)$. Таким образом,

$$
\varphi\left(\left\{q^{1}, q^{2}\right\}, \beta^{l}\right)=\varphi\left(\left\{q^{1}, q^{2}\right\},\left.\beta^{l}\right|_{l-1}, 0\right)=\varphi\left(\left\{q^{n-1}, q^{n}\right\}, 0\right)=\left\{q^{1}, q^{2}\right\}
$$


Занумеруем 2-элементные подмножества множества состояний $Q$ следующим образом:

$$
\begin{aligned}
Q_{1}=\left\{q^{1}, q^{2}\right\}, Q_{2}=\left\{q^{1}, q^{3}\right\}, \ldots, & Q_{n-1}=\left\{q^{1}, q^{n}\right\}, \\
Q_{n} & =\left\{q^{2}, q^{3}\right\}, Q_{n+1}=\left\{q^{2}, q^{4}\right\}, \ldots, Q_{m}=\left\{q^{n-1}, q^{n}\right\},
\end{aligned}
$$

где $m=n(n-1) / 2$. Легко проверить, используя определение функщии переходов, что для любого входного символа $a \in A$, если $\varphi\left(Q_{i}, a\right)=Q_{j}$, то $j-i \leqslant 1$, то есть, подавая произвольный входной символ на пару состояний $Q_{i}$, мы можем продвинуться в смысле введенного нами порядка не более чем на одну позицию вперед в последовательности $Q_{1}, Q_{2}, \ldots, Q_{m}$. Поэтому, поскольку $\varphi\left(Q_{1},\left.\beta^{l}\right|_{l-1}\right)=Q_{m}$, получаем, что

$$
l-1 \geqslant m-1 \Longrightarrow\left|\beta^{l}\right|=l \geqslant \frac{n(n-1)}{2} \text {. }
$$

Так как $\varphi\left(\left\{q^{1}, q^{2}\right\}, \beta^{l}\right)=\left\{q^{1}, q^{2}\right\}$, то $\gamma k$-отличает $q^{1}, q^{2}$, и по предположению индукции

$$
|\gamma| \geqslant n-1+(k-1) \frac{n(n-1)}{2} \text {. }
$$

Итак,

$$
|\alpha|=\left|\beta^{l}\right|+|\gamma| \geqslant \frac{n(n-1)}{2}+n-1+(k-1) \frac{n(n-1)}{2}=n-1+k \frac{n(n-1)}{2},
$$

что и требовалось доказать.

Таким образом, мы доказали, что любое слово $\alpha, k$-отличающее состояния $q^{1}$ и $q^{2}$, имеет длину $|\alpha| \geqslant n-1+(k-1) n(n-1) / 2$. С другой стороны, слово $\alpha=[\beta]^{k-1} \gamma$, где $\beta=\beta_{1} \beta_{2} \ldots \beta_{n-1}, \beta_{i}=(i+1)(i+2) \ldots(n-1) i, i=1,2, \ldots, n-2, \beta_{n-1}=0$, $\gamma=23 \ldots(n-1) 0$, что нетрудно проверить, $k$-отличает состояния $q^{1}, q^{2}$ и имеет длину

$$
\begin{aligned}
|\alpha| & =(k-1)\left(\left|\beta_{1}\right|+\left|\beta_{2}\right|+\ldots+\left|\beta_{n-1}\right|\right)+|\gamma| \\
& =(k-1)((n-1)+(n-2)+\ldots+1)+n-1 \\
& =n-1+(k-1) \frac{n(n-1)}{2}
\end{aligned}
$$

Таким образом, для автомата $\mathfrak{A}$ получаем равенство

$$
L_{\mathfrak{Y}}^{k}\left(q^{1}, q^{2}\right)=n-1+(k-1) \frac{n(n-1)}{2}
$$

и, следовательно,

$$
L(n, k)=n-1+(k-1) \frac{n(n-1)}{2},
$$

что и требовалось доказать.

Сверхсловом в алфавите $A$ назовем всякое отображение $\alpha^{\infty}: \mathbf{N} \rightarrow A$. Это отображение будем записывать в виде бесконечной последовательности символов

$$
\alpha^{\infty}(1) \alpha^{\infty}(2) \ldots \alpha^{\infty}(n) \ldots
$$

Через $A^{\infty}$ обозначим множество всех сверхслов в алфавите $A$. Пусть

$$
\alpha=a(1) a(2) \ldots a(l) \in A^{*}, \quad \beta^{\infty}=b(1) b(2) \ldots b(n) \ldots \in A^{\infty},
$$


тогда полагаем

$$
\begin{aligned}
\alpha \beta^{\infty} & =a(1) a(2) \ldots a(l) b(1) b(2) \ldots b(n) \ldots \\
{[\alpha]^{\infty} } & =\alpha \alpha \ldots \alpha \ldots
\end{aligned}
$$

Доопределим функции $\bar{\varphi}$ и $\bar{\psi}$ произвольного автомата $\mathfrak{U}=(A, Q, B, \varphi, \psi)$ на множестве $Q \times A^{\infty}$ следующим образом. Пусть $\alpha^{\infty}=a(1) a(2) \ldots a(n) \ldots$, тогда

$$
\begin{aligned}
& \bar{\varphi}\left(q, \alpha^{\infty}\right)=\varphi(q, a(1)) \varphi(q, a(1) a(2)) \ldots \varphi(q, a(1) a(2) \ldots a(n)) \ldots \\
& \bar{\psi}\left(q, \alpha^{\infty}\right)=\psi(q, a(1)) \psi(q, a(1) a(2)) \ldots \psi(q, a(1) a(2) \ldots a(n)) \ldots
\end{aligned}
$$

Назовем состояния $q_{1}, q_{2}$ автомата $\mathfrak{A} \infty$-отличимыми сверхсловом $\alpha^{\infty}$, если сверхслова $\bar{\psi}\left(q_{1}, \alpha^{\infty}\right)$ и $\bar{\psi}\left(q_{2}, \alpha^{\infty}\right)$ отличаются в бесконечном числе позиций. Назовем два состояния $\infty$-отличимыми, если существует входное сверхслово, которое их $\infty$-отличает.

Теорема 4. Если состояния $q_{1}, q_{2}$ автомата $\mathfrak{U}$ из $K_{n}(A, B) l$-отличимы $u l>n(n-1) / 2$. то они $\infty$-отличимы.

Доказательство. Действительно, если $q_{1}, q_{2} l$-отличимы, то существует последовательность слов $\alpha_{1}, \alpha_{2}, \ldots, \alpha_{l}$ такая, что для последовательности пар

$$
Q_{1}=\left\{q_{1}, q_{1}^{\prime}\right\}, Q_{2}=\left\{q_{2}, q_{2}{ }^{\prime}\right\}, \ldots, Q_{l+1}=\left\{q_{l+1}, q_{l+1}{ }^{\prime}\right\}
$$

где $q_{i+1}=\varphi\left(q_{i}, \alpha_{i}\right), q_{i+1}{ }^{\prime}=\varphi\left(q_{i}{ }^{\prime}, \alpha_{i}\right)$ верно, что пара состояний $Q_{i}$ отличима входным словом $\alpha_{i}, i=1, \ldots, l$. Поскольку $l>n(n-1) / 2$, существуют натуральные числа $i_{1}$ и $i_{2}, 1 \leqslant i_{1}<i_{2} \leqslant l$, такие, что $Q_{i_{1}}=Q_{i_{2}}$. Тогда сверхслово $\alpha^{\infty}=\beta[\gamma]^{\infty}$, где $\beta=\alpha_{1} \alpha_{2} \ldots \alpha_{i_{1}-1}, \gamma=\alpha_{i_{1}} \alpha_{i_{1}+1} \ldots \alpha_{i_{2}-1}, \infty$-отличает состояния $q_{1}$ и $q_{1}{ }^{\prime}$, что и требовалось доказать.

Теорема 5. Существует автомат $c$ n состояниями, у которого есть два

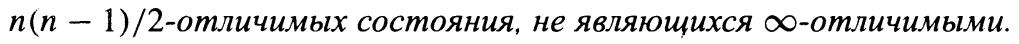

Доказательство. Рассмотрим автомат

$$
\mathfrak{A}=(A, Q, B, \varphi, \psi),
$$

где $Q=\left\{q^{1}, q^{2}, \ldots, q^{n}\right\}, A=\{2,3, \ldots, n-1, \overline{1}, \overline{2}, \ldots, \overline{n-2}\}, B=\{0,1\}$, а функции переходов и выходов определены следуюшим образом:

$$
\begin{aligned}
& \varphi\left(q^{i}, j\right)= \begin{cases}q^{i+1}, & \text { если } i=j, \\
q^{i}, & \text { если } i \neq j, \quad i=1, \ldots, n, \quad j=2, \ldots, n-1,\end{cases} \\
& \varphi\left(q^{i}, \bar{j}\right)= \begin{cases}q^{i+1}, & \text { если } i=j, \\
q^{j+2}, & \text { если } i \neq j, \quad i=1, \ldots, n, \quad j=1, \ldots, n-2,\end{cases} \\
& \psi\left(q^{i}, j\right)= \begin{cases}1, & \text { если } i=j, \\
0, & \text { если } i \neq j, \quad i=1, \ldots, n, \quad=2, \ldots, n-1,\end{cases} \\
& \psi\left(q^{i}, \bar{j}\right)= \begin{cases}1, & \text { если } i=j, \\
0, & \text { если } i \neq j, \quad i=1, \ldots, n, \quad j=1, \ldots, n-2 .\end{cases}
\end{aligned}
$$


Таблица 2.

\begin{tabular}{|c|c|c|c|}
\hline & $\varphi\left(\left\{q^{i}, q^{j}\right\}, a\right)$ & $\psi\left(q^{i}, a\right)$ & $\psi\left(q^{j}, a\right)$ \\
\hline$a=i$ & $\left\{q^{i+1}, q^{j}\right\}$ & 1 & 0 \\
\hline$a=j$ & $\left\{q^{i}, q^{j+1}\right\}$ & 0 & 1 \\
\hline$a=k(k \neq i, j)$ & $\left\{q^{i}, q^{j}\right\}$ & 0 & 0 \\
\hline$a=\bar{i}$ & $\left\{q^{i+1}, q^{j+2}\right\}$ & 1 & 0 \\
\hline$a=\bar{j}$ & $\left\{q^{j+1}, q^{j+2}\right\}$ & 0 & 1 \\
\hline$a=\bar{k}(k \neq i, j)$ & $\left\{q^{k+2}\right\}$ & 0 & 0 \\
\hline
\end{tabular}

Упорядочим неупорядоченные пары состояний автомата $\mathfrak{U}$ :

$$
Q_{1}=\left\{q^{1}, q^{2}\right\}, Q_{2}=\left\{q^{1}, q^{3}\right\}, \ldots, Q_{n-1}=\left\{q^{1}, q^{n}\right\}, Q_{n}=\left\{q^{2}, q^{3}\right\}, \ldots, Q_{l}=\left\{q^{n-1}, q^{n}\right\}
$$

Положим $l=n(n-1) / 2$. Рассмотрим таблицу 2 .

Из нее легко видеть, что для любого входного символа $a$ и пары состояний $Q_{m}=\left\{q^{i}, q^{j}\right\}$, если $\varphi\left(Q_{m}, a\right)=Q_{p}$, то $m \leqslant p$ и если $\psi\left(q^{i}, a\right) \neq \psi\left(q^{j}, a\right)$, то $m<p$. Поэтому, если слово $\alpha$ отличает пару состояний $Q_{m}$ и $\varphi\left(Q_{m}, \alpha\right)=Q_{p}$, то $m<p$. Допустим, что пара состояний $Q_{1}=\left\{q^{1}, q^{2}\right\} \infty$-отличима сверхсловом $\alpha^{\infty}=\alpha_{1} \alpha_{2} \ldots \alpha_{j} \ldots$, где $\alpha_{j}$ отличает $Q_{i_{j-1}}=\varphi\left(Q_{1}, \alpha_{1} \alpha_{2} \ldots \alpha_{j-1}\right)$ и переводит в $Q_{i_{j}}=\varphi\left(Q_{i_{j-1}}, \alpha_{j}\right)$. Получаем бесконечную последовательность пар состояний

$$
Q_{1}=Q_{i_{0}}, Q_{i_{1}}, Q_{i_{2}}, \ldots, Q_{i_{j}}, \ldots
$$

и используя доказанное выше, получаем бесконечно возрастающую последовательность индексов $i_{0}<i_{1}<i_{2}<\ldots<i_{j}<\ldots$, чего быть не может, так как $i_{j} \leqslant l, j \in \mathbf{N}$. Значит, пара состояний $Q_{1}$ не $\infty$-отличима. Однако она $n(n-1) / 2$-отличима словом

$$
\alpha=23 \ldots(n-1) \overline{1} 34 \ldots(n-1) \overline{2} 45 \ldots(n-1) \ldots \overline{(n-2)}(n-1) .
$$

Таким образом, автомат $\mathfrak{U}$ обладает требуемыми свойствами, что и требовалось доказать.

Заметим, что все ранее вводимые отношения $R$ на $B$ были рефлексивными. Рассмотрим случай, когда $R$ есть отношение неравенства, то есть соотношение $x R y$ равносильно неравенству $x \neq y$. Таким образом, состояния $q_{1}, q_{2}$ автомата $\mathfrak{A}$ являются $(\neq)$-отличимыми словом $\alpha$, если $\psi\left(q_{1}, \alpha\right)=\psi\left(q_{2}, \alpha\right)$.

Теорема 6. Справедливо равенство

$$
L_{\neq}(n)=\frac{n(n-1)}{2}+1
$$

Доказательство. Свяжем с автоматом $\mathfrak{U}=(A, Q, B, \varphi, \psi)$ автомат без выхода $\mathfrak{U}^{\prime}=\left(A, Q, Q, \varphi, \psi^{\prime}\right)$, где $\psi^{\prime}(q, a) \equiv q$. Очевидно, что если состояния $q_{1}, q_{2}$ в автомате $\mathfrak{U}^{\prime}(\neq)$-отличимы словом $\alpha$, то они $(\neq)$-отличимы им в автомате $\mathfrak{A}$. Поэтому

$$
L_{\mathfrak{T}, \neq}\left(q_{1}, q_{2}\right) \leqslant L_{\mathfrak{P I}^{\prime}, \neq}\left(q_{1}, q_{2}\right)
$$

и для определения величины $L_{\neq}(n)$ достаточно ограничиться автоматами без выхода. 
Рассмотрим произвольный такой автомат $\mathcal{A}$. Пусть $\alpha^{l}$ - минимальное по длине входное слово, $(\neq)$-отличающее его состояния $q_{1}, q_{2}$. Тогда $\left.\alpha^{l}\right|_{l-1}$ есть минимальное по длине слово, склеивающее эти состояния, и мы получаем, что $L_{\neq}(n)=L_{S}(n)+1$, где $L_{S}(n)-$ аналогичная функщия Шеннона для минимальной длины склеивающего слова. Поэтому достаточно показать, что $L_{S}(n)=n(n-1) / 2$.

Действительно, пусть $\alpha=a(1) a(2) \ldots a(l)$ - кратчайшее слово, склеивающее состояния $q_{1}, q_{2}$ автомата $\mathfrak{U} \in K_{n}(A, B)$ и

$$
Q_{i}=\varphi\left(\left\{q_{1}, q_{2}\right\}, a(1) a(2) \ldots a(i)\right), \quad 0 \leqslant i \leqslant l .
$$

В последовательности $Q_{0}, Q_{1}, \ldots, Q_{l-1}$ не может быть двух одинаковых элементов $Q_{i}=Q_{j}, i<j$, поскольку тогда слово

$$
\beta=a(1) a(2) \ldots a(i) a(j+1) a(j+2) \ldots a(l-1)
$$

также склеивает состояния $q_{1}$ и $q_{2}$ и имеет длину $|\beta|<|\alpha|$, что невозможно в силу свойств $\alpha$. Следовательно, $l \leqslant n(n-1) / 2$. С другой стороны, как показывает пример, приведенный при доказательстве теоремы 1 , существует автомат с $n$ состояниями, у которого есть два состояния $q^{0}$ и $q^{|n / 2|}$ с минимальной длиной склеивающего слова, равной $n(n-1) / 2$. Лемма доказана.

Автор выражает признательность своему научному руководителю А. С. Подколзину и В. Б. Кудрявцеву за помощь во время работы над статьей.

\section{Список литературы}

1. Мур Э. Ф., Умозрительные эксперименты с последовательными машинами. В кн.: Aвтоматы. ИЛ, Москва, 1956, с. 179-210.

2. Кудрявцев В. Б., Подколзин А. С., Ушчумлич Ш. М., Введение в теорию абстрактиых автоматов. Изд-во МГУ, Москва, 1985.

3. Кудрявцев В. Б., Алешин С. В., Подколзин А. С., Элемеиты теории автоматов. Изд-во МГУ, Москва, 1978.

Статья поступила 11.10.2002. 